\title{
Age trends in recognition memory for pictures: The effects of delay and testing procedure
}

\author{
FREDERICK J. MORRISON \\ University of Minnesota, Minneapolis, Minnesota 55455 \\ MARSHALL M. HAITH \\ University of Denver, Denver, Colorado 80210 \\ and \\ JEROME KAGAN \\ Harvard University, Cambridge, Massachusetts 02138
}

\begin{abstract}
Experiment 1 examined the effects of three delay intervals $(0,24$, and $48 \mathrm{~h})$ on recognition memory performance of 5-, 8-, and 11-year-olds and adults using a forced-choice procedure. Results revealed significant drops in performance after $24 \mathrm{~h}$ for 5-year-olds and after $48 \mathrm{~h}$ for 8-year-olds, with no performance decrement for older age groups. However, observations made during the first experiment raised the possibility that performance in the forced-choice procedure may have been biased, since subjects could infer which item was presented previously from high confidence that the new item had never been presented. Since age differences in use of this inferential process may have exaggerated the performance differences observed with the forced-choice procedure, Experiment 2 employed a single-item yes-no testing procedure to assess age and delay effects on recognition memory. Performance increased with age and decreased with delay, but no differential performance decrement occurred across delay as a function of age. Together, results confirmed earlier studies in demonstrating age invariance in information loss across time.
\end{abstract}

Memory development has been the focus of extensive investigation over the past decade. Of the two procedures typically used to assess memory performance, recall and recognition, more is known about the factors influencing the growth of recall memory (see Kail \& Hagen, 1977, for a review). With regard to recognition memory, it has been shown that recognition performance does typically increase with age (Mandler \& Stein, 1974; Nelson \& Kosslyn, 1976; Perlmutter \& Myers, 1974; Rogoff, Newcombe, \& Kagan, 1974; Tversky \& Teiffer, 1976), although recognition accuracy is quite high, even in preschool children (Brown \& Scott, 1971; Corsini, Jacobus, \& Leonard, 1969; Perlmutter \& Myers, 1976). Increasing the similarity between targets and distractors has been shown to increase the size of developmental differences in recognition memory (Dirks \& Neisser, 1977; Mandler \& Stein, 1974; Tversky \& Teiffer, 1976). In addition, degree of organization in a pictorial scene affected age differences found in recognition accuracy (Mandler \& Robinson, 1978). First- through fifth-grade children performed much worse on unorga-

This research was supported by U.S. Public Health Grant HD02680 to Marshall Haith and by Research Scientist Development Award MH 00226-01 to Frederick J. Morrison. Requests for reprints should be sent to Frederick J. Morrison, Institute of Child Development, University of Minnesota, Minneapolis, Minnesota 55455. nized pictures than they did on pictures of objects organized in a real-world scene. Adults, in contrast, did not differ in recognition accuracy for organized vs. unorganized scenes (Mandler \& Ritchey, 1977). One potentially important variable is the length of delay between inspection and testing. If loss of information over time varies across age, developmental differences in recognition memory should vary as a function of delay interval. Fajnsztejn-Pollack (1973) compared recognition performance for 280 pictures in children 5-16 years old after delay intervals of $2,5,10,20$, and 49 weeks following initial inspection. Although recognition performance declined across the delay, no age differences were found in the rate at which items were lost from memory over time. However, FajnsztejnPollack's earliest assessment of delayed recognition performance was fully 2 weeks following initial presentation, during which time age differences in rate of decay might have existed. In this regard, Rogoff et al. (1974) found significant decreases in recognition performance over a 1-week interval for 4-, 6-, and 8-year-old children, with no differential decay across age. Since they did not include samples of older children or adults, the developmental significance of poorer recognition across delay is unclear. A similar finding of decreasing recognition accuracy in preschoolers across a 28-day delay was obtained by Brown and Scott (1971), but 
again, no older age groups were tested. Thus, the question of possible age differences in rate of information loss from memory remains unanswered. The present study attempted to compare recognition memory performance across a wide age range ( 5 years through adulthood) following delay intervals of 0,24 , or $48 \mathrm{~h}$.

\section{EXPERIMENT 1: AGE DIFFERENCES IN RECOGNITION MEMORY IN A FORCED-CHOICE PARADIGM}

\section{Method}

A total of 72 subjects from four age groups (5-year-olds, 8-year-olds, 11-year-olds, and college-aged adults) was used in this experiment.

The stimuli were 120 chromatic pictures cut out of magazines and pasted on $5 \times 7$ in. white cardboard cards. Pictures depicted a single, salient object (e.g., shoe, truck, fruit) with as little extraneous context as possible. Sixty pictures, randomly chosen, served as presentation stimuli; the remaining 60 pictures were used as comparison stimuli during the testing phase. A set of 10 warm-up cards (five presentation and five comparison pictures) was constructed for use prior to the experimental phase of the experiment.

The experiment was conducted in three phases. (1) Warmup phase: A series of five warm-up cards was presented, and the subject was told to look at each picture carefully one at a time. After the subject had proceeded through the pictures self-paced, the experimenter took the five inspection pictures and paired them (out of sight of the subject) with five other pictures. Each pair of pictures was then shown to the subject, and he/she was instructed to point to the picture that had been shown before. (2) Inspection phase: The subject was told that he/she was going to look at a large number of pictures and would be asked to remember them either immediately or $24 \mathrm{~h}$ or $48 \mathrm{~h}$ after seeing the pictures. Care was taken to emphasize to the children the length of time involved, such as by telling them what day they would be tested and how many nights they would sleep before returning. The subject then proceeded self-paced through the 60 pictures. Each subject was presented one of six random orders of the 60 pictures. To control for list-specific effects, half of the subjects viewed one set of 60 pictures during the inspection phase, and the other half saw a second set. (3) Testing phase: The experimenter showed the subject two pictures, one from the inspection series and the other a new picture not previously presented. The subject's task was to point to the picture that had been presented in the previous phase. Position of the correct picture (left or right) was counterbalanced evenly across the 60 pairs of pictures. For any given subject, the order in which the pictures was tested was identical to the order of presentation. Within each age group, subjects were divided into three separate delay conditions: 0 delay, in which the testing phase occurred immediately after the inspection phase; 24-h delay, in which testing took place the following day (as close as possible to $24 \mathrm{~h}$ ); 48-h delay, in which testing occurred 2 days following inspection. The complete experiment took between 20 and $30 \mathrm{~min}$.

\section{Results and Discussion}

A two-way analysis of variance was performed on the mean number of errors ${ }^{1}$ made on the 60 test pairs, with age (5-year-olds, 8-year-olds, 11-year-olds, and adults) and delay $(0,24$, and $48 \mathrm{~h})$ as the major independent variables. Number of errors committed varied significantly across age $[F(3,60)=9.01]$. Mean number of errors varied from 6.6 for the 5-year-olds to $3.5,2.2$, and 1.5 for the 8-year-olds, 11-year-olds, and adults, respectively.
Amount of delay also produced reliable differences in performance $[F(2,60)=4.48]$. Number of errors ranged from 1.96 at 0 delay to 3.83 and 4.63 at 24 and $48 \mathrm{~h}$, respectively. A reliable Age by Delay interaction was found $[F(6,60)=3 \cdot 71]$. As shown in Figure 1, individual comparisons among means revealed that performance of 5-year-olds dropped sharply between 0 - and 24-h delay $[\mathrm{t}(60)=18.1, \mathrm{p}<.001]$, with no further decrease in performance at $48 \mathrm{~h}$. Eight-year-olds showed little drop in performance after $24 \mathrm{~h}$, but a reliable decrease in recognition following 48 -h delay $[\mathrm{t}(60)=10.6, \mathrm{p}<.001$, for comparison between 0 - and 48-h delays]. The two older age groups did not show significant decreases in recognition performance over the 48-h delay interval.

Results showed clear age differences in recognition memory performance, replicating earlier studies (Mandler \& Robinson, 1978; Nelson \& Kosslyn, 1976; Rogoff et al., 1974). More important, findings for the delay factor revealed age differences in rate of decay of information over time. Performance of 5-year-olds indicated that significant amounts of information were lost in the first $24 \mathrm{~h}$ following inspection, with little drop thereafter. Eight-year-olds appeared not to lose significant amounts of information until after a 48-h delay. Older groups did not appear to lose much information at all.

Although results of this experiment corroborated the hypothesis of age differences in rate of decay of information over time, observations made during testing raised the possibility that the forced-choice procedure introduced some biases that could have affected the results. Specifically, several adult subjects commented spontaneously that they often decided which of the two pictures had been presented previously not by actually

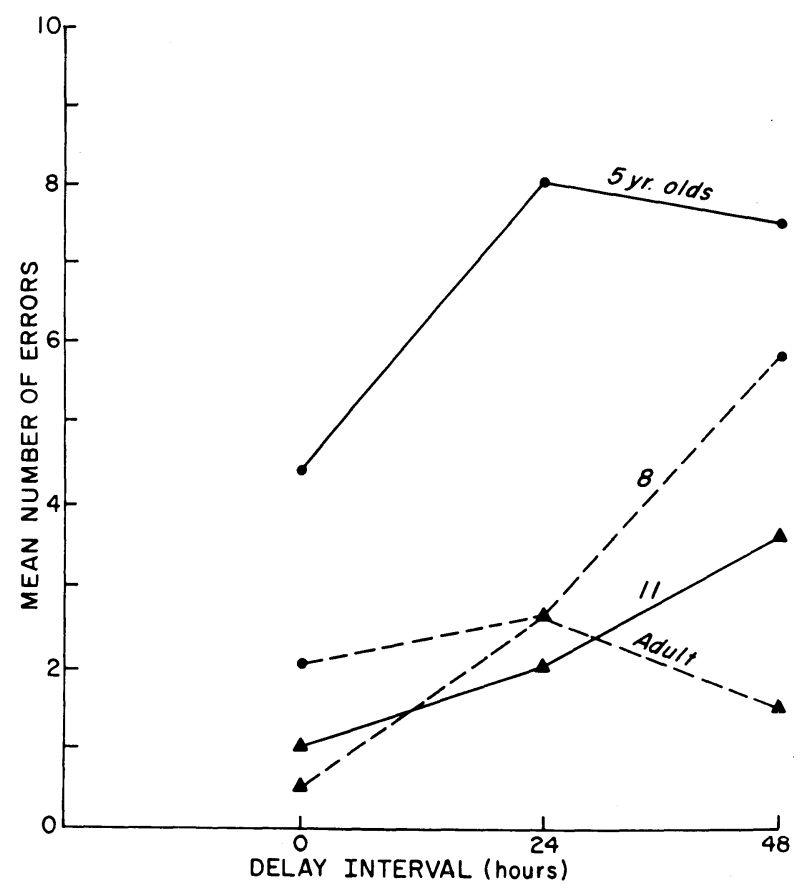

Figure 1. Mean number of errors made by the four age groups in Experiment 1 as a function of delay interval. 
recognizing the old picture, but as a result of being very certain that the new picture had never been presented. Hence, the forced-choice procedure may not only directly assess recognition of items actually stored in memory, but may also assess how well a subject can infer what was the previously presented item from knowledge that a new item was never shown. Increases with age in the tendency or ability to utilize these inferential processes, as well as increased probability of use of these inferences with delay, might have resulted in an inaccurate picture of the nature of age and delay effects in recognition memory performance. To investigate this possibility, a second experiment was conducted that employed a single-item yes-no procedure. This procedure minimized the effectiveness of inferential processes, and it allowed a separate assessment of performance on old and new items.

\section{EXPERIMENT 2: AGE DIFFERENCES IN RECOGNITION MEMORY IN A YES-NO PARADIGM}

\section{Method}

A different group of 54 subjects from three age groups (5-year-olds, 8-year-olds, and adults) was tested. The same set of 120 chromatic pictures used in Experiment 1 was used. The inspection phase of this experiment was identical to the previous experiment. During the testing phase (as well as test trials in the warm-up phase), subjects were shown pictures one at a time and were required to respond "yes" or "no," depending on whether they thought they had seen the picture during the inspection phase. The set of 60 pictures shown during the inspection phase (old) was combined with another set of 60 pictures not presented (new). The ordering of pictures was random, with the restriction that an equal number of old and new pictures be presented every 20 trials and that no more than three old or new pictures appear on consecutive test trials. Thus, a total of 120 pictures was tested. As in Experiment 1, subjects were divided equally into $0-, 24-$, and $48-\mathrm{h}$ delay conditions. The entire experiment lasted approximately $30-40 \mathrm{~min}$.

\section{Results and Discussion}

A three-way analysis of variance was performed on number of errors made, with age (5-year-old, 8-yearolds, and adults) and delay (0,24, and $48 \mathrm{~h})$ as betweensubjects variables and familiarity (old vs. new pictures) as a within-subjects variable. A significant age effect $[F(2,45)=6.34]$, revealed that adults performed significantly better than 8-year-olds $[\mathrm{t}(45)=8.71$, who performed significantly better than 5-year-olds [t(45) = $7.73]$. A significant delay effect $[F(2,45)=4.41]$ indicated that recognition performance decreased with increases in delay interval. No Age by Delay interaction was observed (see Table 1). More errors were made on old pictures (7.5) than on new pictures $(3.1)[F(1,45)=$ 45.6]. This difference was qualified by a significant Age by Familiarity interaction $[F(2,45)=6.7]$, shown in Figure 2 . As can be seen, age differences were strongest on old items, with both 5-year-olds and 8-year-olds making more errors than adults $[\mathrm{t}(45)=19.6$ and 18.6 , respectively, for 5-year-olds and 8-year-olds when compared with adults]. In contrast, errors on new items were much
Table 1

Mean Number of Errors Made by the Three Age Groups in Experiment 2 at the Three Delay Intervals

\begin{tabular}{cccc}
\hline & \multicolumn{3}{c}{ Age } \\
\cline { 2 - 4 } Delay & 5 Years & 8 Years & Adult \\
\hline 0 & 11.7 & 12.7 & 3.2 \\
24 & 18.7 & 7.3 & 6.7 \\
48 & 14.5 & 14.0 & 6.8 \\
\hline
\end{tabular}

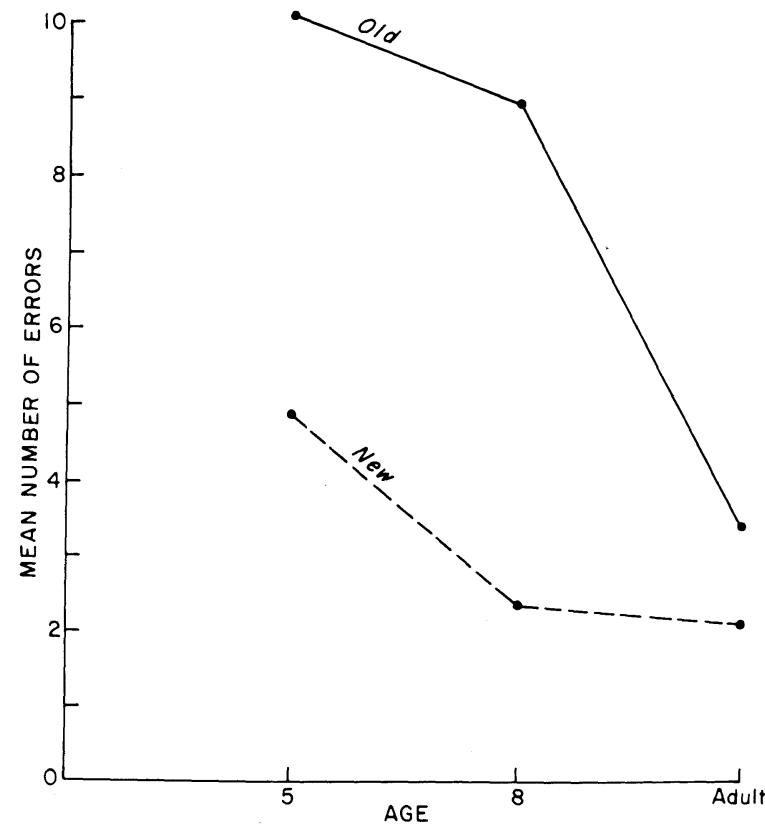

Figure 2. Mean number of errors made by the three age groups in Experiment 2 on old and new pictures.

lower, although 5 -year-olds did make significantly more errors than the two older age groups $[\mathrm{t}(45)=5.1$ and 5.8 , respectively, for comparisons with 8-year-olds and adults]. No other interactions approached significance.

In an attempt to compare directly performance in the forced-choice vs. yes-no procedure across age, individual error scores were converted to percentages and subjected to an analysis of variance, with age and testing procedure as between-subjects variables. A significant age effect $[F(2,90)=15.63]$ and a significant condition effect $[F(1,90)=3.96]$ were qualified by a reliable interaction between age and testing condition $[F(2,90)=3.88]$. Shown in Table 2, this interaction reflected the fact that testing condition did not significantly alter performance for the 5-year-olds $[t(90)=$ 1.12]. In contrast, for 8-year-olds and adults, performance in the forced-choice procedure was reliably better than in the yes-no procedure $[\mathrm{t}(90)=8.11$ and 6.40 , respectively, for 8-year-olds and adults] .

In summary, results from Experiment 2 revealed increases with age in recognition memory performance and decreases with delay in recognition accuracy. However, in contrast to the results of Experiment 1, 
Table 2

Mean Percent Errors Committed by the Three Age Groups in the Forced-Choice and Yes-No Procedures

\begin{tabular}{lccc}
\hline & \multicolumn{3}{c}{ Age } \\
\cline { 2 - 4 } Procedure & 5 Years & 8 Years & Adult \\
\hline Forced Choice & 11.0 & 5.8 & 2.6 \\
Yes-No & 12.5 & 9.5 & 4.6 \\
\hline
\end{tabular}

no differential drop in performance was observed as a function of age. In addition, major age differences occurred primarily on items previously presented, with adults recognizing old items much better than the two younger groups. In contrast, performance on new items was much higher than on old items, although 5-year. olds did not perform as well on new items as did the two older groups. Finally, comparison of performance levels in Experiments 1 and 2 revealed that 8-year-olds and adults performed much better in the forced-choice procedure than in the yes-no procedure, whereas 5-yearolds showed no difference across the two procedures.

The original purpose of the present research was to investigate the effect of delay on recognition memory across age. Results from the first experiment supported the notion that younger children (5-and 8-year-olds) lost greater amounts of information over time than did older children and adults. However, the possibility that the results might be due not to age differences in memory processes, but to differences in inferential processes led to utilization of a yes-no procedure in the second experiment. Findings for performance on old vs. new items in that study confirmed the suspicion that subjects knew with a high degree of accuracy which items had not been presented before. Performance on new items was higher than on old items. When performance levels were compared across the two testing procedures, the older subjects performed reliably higher in the forced-choice than in the yes-no procedure, whereas younger subjects showed no difference in performance in the two procedures. These findings are consistent with the interpretation that older subjects took advantage of the inferential possibilities afforded by the forced-choice procedure, but the youngest children did not. Taken together, results from the two experiments raise the possibility that the differential rate of decay of information across age noted in the first experiment might be due to age differences in the tendency to utilize inferential processes to decide which picture had been presented. It is possible, for example, that at 0 delay all age groups had sufficient information stored in memory to simply match to old items presented during testing. However, as information decayed over time and subjects became less certain whether an old item had been presented, older children and adults may have begun to rely more on their stronger knowledge of not having seen one of the items to infer that they must have seen the other item. Younger children, in turn, may not have used this inferential process but may have continued to rely on the strength of the match between the old item and the memory trace. The fact that the youngest children did not perform as well on new items as did the older age groups is consistent with the notion that they relied less on the new item as a basis for judging which item had been presented.

With regard to the original question of possible age differences in rate of decay of information in memory, it is suggested that rate of decay of information is invariant across age and that age differences in rate of decay found in Experiment 1 are better explained as age differences in the tendency to use inferential processes in a forced-choice recognition procedure.

\section{REFERENCES}

Brown, A. L., ScotT, M. S. Recognition memory for pictures in preschool children. Journal of Experimental Child Psychology, 1971, 11, 401-412.

Corsini, D. A., J Acobus, K. A., \& Leonard, S. D. Recognition memory of preschool children for pictures and words. Psychonomic Science, 1969, 16, 192-193.

Dirks, J., \& Neisser, U. Memory for objects in real scenes: The development of recognition and recall. Journal of Experimental Child Psychology, 1977, 23, 315-328.

Fajnsztejn-Pollack, G. A developmental study of decay rate in long-term memory. Journal of Experimental Child Psychology, $1973,16,225-235$.

KaIl, R. V., \& Hagen, J. W. Perspectives on the development of memory and cognition. Hillsdale, N.J: Erlbaum, 1977.

Mandle R, J. M., \& Ritchey, G. H. Long-term memory for pictures. Journal of Experimental Psychology: Human Learning and Memory, 1977, 3, 386-396.

Mandle R, J. M., \& Robinson, C. R. Developmental changes in picture recognition. Journal of Experimental Child Psychology, 1978, 26, 122-136.

Mandle R, J. M., \& STEin, N. L. Recall and recognition of pictures by children as a function of distractor similarity. Journal of Experimental Psychology, 1974, 102, 657-669.

Nelson, K. E., \& Kosslyn, S. M. Recognition of previously labelled or unlabelled pictures by 5 -year-olds and adults. Journal of Experimental Child Psychology, 1976, 21, 40-45.

Perlmutter, M., \& Myers, N. A. Recognition memory development in two- to four-year-olds. Developmental Psychology, 1974, 10, 447-450.

Perlmutter, M., \& Myers, N. A. Recognition memory in preschool children. Developmental Psychology, 1976, 12, 271-272.

Rogoff, B., Newcombe, N., \& Kagan, J. Planfulness and recognition memory. Child Development, 1974, 45, 972-977.

Tversky, B., \& Teiffer, E. Development of strategies for recall and recognition. Developmental Psychology, 1976, 12, 406-410.

\section{NOTE}

1. In addition to analyses of raw error data, scores were converted to $\mathrm{d}^{\prime}$ scores and subjected to analyses of variance. In no instance did the results from the analysis of $\mathrm{d}^{\prime}$ scores differ from those obtained in the analysis of error scores. Thus, for simplicity of exposition, only results from the analysis of error scores will be reported her.

(Received for publication August 5, 1980.) 